\title{
Action potential alterations induced by single F11 neuronal cell loading
}

\author{
Miren Tamayo-Elizalde ${ }^{a}$, Haoyu Chen $^{a}$, Majid Malboubi ${ }^{a, b}$, Hua Ye $^{a}$ and Antoine Jerusalem ${ }^{a, *}$ \\ ${ }^{a}$ Department of Engineering Science, University of Oxford, Oxford, UK \\ ${ }^{b}$ Currently: Department of Mechanical Engineering, University of Birmingham, Birmingham, UK
}

\section{ARTICLE INFO}

\section{Keywords:}

Neuron multiphysics

Action potential

Mechanoelectrical coupling

\begin{abstract}
A B S T R AC T
Several research programmes have demonstrated how Transcranial Ultrasound Stimulation (TUS) can non-invasively and reversibly mechanically perturb neuronal functions. However, the mechanisms through which such reversible and a priori non-damaging behaviour can be observed remain largely unknown. While several TUS protocols have demonstrated motor and behavioural alterations in in vivo models, in vitro studies remain scarce. In particular, an experimental framework able to load mechanically an individual neuron in a controlled manner and simultaneously measure the generation and propagation of action potentials before, during and after such load, while allowing for direct microscopy, has not been successfully proposed. To this end, we herein present a multiphysics setup combining nanoindentation and patch clamp systems, assembled in an inverted microscope for simultaneous bright-field or fluorescence imaging. We evaluate the potential of the platform with a set of experiments in which single dorsal root ganglion-derived neuronal cell bodies are compressed while their spontaneous activity is recorded. We show that these transient quasi-static mechanical loads reversibly affect the amplitude and rate of change of the neuronal action potentials, which are smaller and slower upon indentation, while irreversibly altering other features. The ability to simultaneously image, mechanically and electrically manipulate and record single cells in a perturbed mechanical environment makes this system particularly suitable for studying the multiphysics of the brain at cell level.
\end{abstract}

\section{Introduction}

Similar to the way general anaesthetics have evolved as a cornerstone of modern surgery, Transcranial Ultrasound Stimulation (TUS) is poised to become a significant part of the future of brain clinical intervention [1]. In both cases, the neuronal membrane appears to play a major role in the way their actions occur. In particular, it is increasingly clear that the wide array of functions it is involved in, at the interface of mechanics, biochemistry and electrophysiology, hints at a concurrent multiphysics role whereby any alteration of a physical property (e.g., membrane potential, $\mathrm{pH}$, mechanical deformation, etc.) simultaneously affects the other physics.

Traditionally, however, the neuronal action potential (AP) is often solely regarded as an electrochemical signal [2], despite increasing evidence of thermodynamical changes accompanying the AP [1]. In particular, a neuronal membrane displacement has been observed to accompany the electrophysiological changes occurring during AP propagation [3-5]. These findings have been possible, to a large extent, thanks to the development of novel experimental techniques whose sensitivities enable the detection of nanometer-scale membrane deflections. While highly desirable to further identify and understand the mechanisms at play in multiphysics phenomena such as TUS, simultaneously measuring and monitoring them at the cellular level remains challenging.

Atomic Force Microscopy (AFM) and patch clamp are extensively used techniques when studying single cell mechanics and electrophysiology, respectively. However, their

*To whom correspondence should be addressed. E-mail: antoine.jerusalem@eng.ox.ac.uk combination is not straightforward, as standard AFM sample stages do not generally allow room for conventional patch clamp systems. As a result, while no commercial system is available, different research groups have built customised combined setups. A modified AFM with an adapted scan head to allow clearance for patch clamp pipettes, compatible with an inverted microscope, was introduced in Ref. [6]. Similarly, a customised AFM/whole-cell voltage clamp system was proposed for the investigation of membrane electromotility in voltage-clamped HEK-293 cells [7, 8]. Planar patch clamp systems have also been successfully combined with AFMs [9-11]. Furthermore, microchannelled AFM cantilevers have permitted simultaneous mechanical and electrical measurements [12]. Open-loop systems, mainly based on piezoelectric actuators that push glass probes [1315] or pillar arrays [16] into cells, have been widely used to study mechanically activated currents in sensory neurons, recorded with patch clamp amplifiers. However, in these experiments, the imposed load or displacement was indirectly calculated, with no feedback control available. It must be noted that a closed-loop force-clamp system has been combined with patch clamp but at larger scale, to investigate touch sensation in C-elegans [17]. As such, a feedback-loop framework to investigate the effects of a mechanical load on the spontaneous activity of single cells with simultaneous microscopy has not yet been proposed.

To this end, we present here a multiphysics setup that simultaneously combines nanoindentation and patch clamp in an inverted microscope for concurrent bright-field or fluorescence imaging. A set of experiments is designed to quantify the influence of mechanical loading on the generation and propagation of neuronal APs. In particular, the multiphysics setup, presented in Section 2, is used for the measurement 
of spontaneous APs alteration induced by quasi-static indentation in Section 3, leading to a reversible decrease of AP amplitude and rate of change during loading, but an irreversible hyperpolarisation, among other AP features. To investigate the mechanisms behind such alterations, a study making use of a finite element (FE) simulation of the 3D indentation coupled to an electrophysiologically based model is conducted in Section 4. This analysis and the accompanying discussion conclude that the observed response cannot be solely explained by a membrane area change, thus pointing towards a coupled effect of the membrane deformation and ion channel action alteration.

\section{Materials and Methods}

\subsection{Experimental methods}

\subsubsection{Cell culture}

F11 cells (ECACC, UK), a hybrid between mouse N18TG-2 neuroblastoma and embryonic rat dorsal root ganglion (DRG) neurons, and commonly used as a functional DRG neuronal model [18], were chosen for these experiments for their mature neuronal features such as outgrowth of extensive neurite-like processes and spontaneous APs [19].

F11s were expanded and differentiated following published protocols [20] (details in Section 7). $\sim 5 \times 10^{4}$ cells were seeded per glass bottom dish (WillCoWells) and differentiated for five days [18] prior to experiments, topping up the media every two days. The low confluence of cells seeded for differentiation was empirically optimised in order to obtain sparsely distributed, yet connected, cells, see Figure 1. Higher seeding densities resulted in less and shorter neurites, as well as cell bodies in contact, preventing single cell indentation studies.

\subsubsection{Inverted microscope}

The arrangement of the setup allows for a continuous inspection and control of the nanoindenter probe and patch pipette positions via bright-field imaging through an inverted Nikon Eclipse Ti microscope (see Figure 1, left). Fluorescence (both widefield and confocal) imaging is also available throughout the experiment, with the advantage that the probes are hidden from the field of view (see Figure 1, right).

\subsubsection{Mechanics}

Cell indentations were carried out with the Chiaro nanoindenter (Optics11). Probes of $3 \mu \mathrm{m}$ spherical tip radius and $0.05 \mathrm{~N} / \mathrm{m}$ cantilever stiffness were used.

In order to avoid cell damage during loading, such as cytoskeletal components rupture [21], the quasi-static indentation depth was chosen to be within the cell's linear viscoelastic region (LVR). F11's LVR was determined by subsequently indenting $\mathrm{N}=9$ cell bodies at increasing depths of 2,3 and $4 \mu \mathrm{m}$ for $20 \mathrm{~s}$ (that ensured full relaxation) and by fitting and comparing the corresponding Young's moduli (see Section 7).
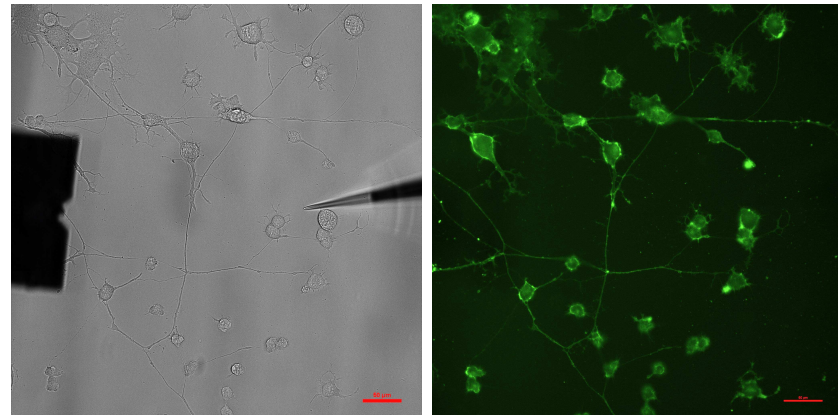

Figure 1: Brightfield and widefield fluorescence images of differentiated F11 cells on a glass bottom dish. Nanoindenter and pipette do not obstruct vision in fluorescence images. Scale bars are $50 \mu \mathrm{m}$.

The typical relaxation times of F11 cells were studied via stress-relaxation tests. In a stress-relaxation experiment, the stress (or force $F$, as measured by the nanoindenter) exhibits an exponentially decaying response. By fitting this decay, the characteristic relaxation times corresponding to different viscoelastic relaxations mechanisms can be identified. Single and double exponential decays were used to fit the stress-relaxation curves from constant strain $(2 \mu \mathrm{m}$ indentation depth) indentations in $\mathrm{N}=13 \mathrm{~F} 11$ cell bodies, see Appendix.

The viscoelastic behaviour of F11 cell bodies was further studied through dynamic mechanical analysis (DMA) experiments, imposing constant oscillating amplitudes of 500 $\mathrm{nm}$ around a mean of $2 \mu \mathrm{m}$, at five different frequencies: $0.1,0.316,1,3.16$ and $10 \mathrm{~Hz}$ in $\mathrm{N}=7 \mathrm{~F} 11$ cell bodies. The cells' storage and loss moduli at each frequency were calculated by fitting their force-indentation responses (details in Section 7).

\subsubsection{Electrophysiology}

F11s on their fifth and sixth differentiation days were used for experiments, with passages ranging from 14 to 23 . Neuronal maturation was confirmed by immunohistochemistry with NeuN marker (Abcam, ab177487). Healthy F11s with sufficiently large round somas were selected for the study. While high cell density and well-connected neurons are desired for high spontaneous spiking rates, relative isolation of the cell is required to minimise the mechanical influence of neighbouring cells during indentation. As a compromise, cells whose somas were not in direct contact with those of other neurons, but still connected to other cells through their neurites, were chosen for the experiments.

Pipettes with resistances between 2 and $5 \mathrm{M} \Omega$, as measured in the bath solution, were pulled from borosilicate glass capillaries (BF100-78-10, Sutter Instruments) through a $2.5 \times 2.5 \mathrm{~mm}^{2}$ box filament with a P-1000 puller (Sutter Instruments), following optimised parameters from Ref. [22]. Compositions of intra- and extracellular solutions can be found in Section 7. The liquid junction potential between the solutions was experimentally determined (to $-11.9 \mathrm{mV}$ ), and the membrane potential values were corrected accord- 

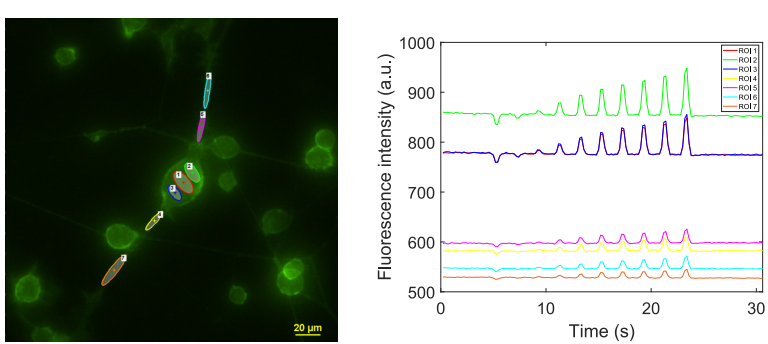

Figure 2: The voltage of all cell membrane regions follows a voltage clamp episodic stimulation of 10 sweeps of $20 \mathrm{mV}$ voltage steps, starting at $-100 \mathrm{mV}$. On the right, the fluorescent intensities (FluoVolt Membrane Potential kit, ThermoFisher) of the regions selected on the left. Scale bar is $20 \mu \mathrm{m}$.

ingly.

Prior to performing the indentation experiments, a space clamp test was performed to confirm the whole cell spacial extension of the clamp. As the distance nanoindentermicropipette is maximised within the soma, the influence of local mechanical deformation on the measured current could potentially be underestimated in case of poor space-clamp, i.e., in case of excessive gradient of voltage within the cell. To this end, the FluoVolt Membrane Potential Kit (Thermofisher) fluorescent dye was used during a voltage clamp experiment. The voltage clamp was started at $-100 \mathrm{mV}$ and increased by 10 voltage steps of $20 \mathrm{mV}$ amplitude. Fluorescence intensity, indicator of membrane voltage values, was quantified during hyperpolarisation and depolarisation steps in the soma and proximal regions of the neurites, see Figure 2. Results show that, even though a negative gradient in the voltage amplitude is observed, the activity remains the same in the soma within $10 \%$, and ionic currents occur, in phase, for all analysed regions. This ensures that the electrical alterations arising from the indented region are captured by the patch electrode.

The cells' spontaneous activity was recorded in gap-free current clamp (I0) mode. To minimise variability introduced by step-wise episodic stimulation, only cells showing spontaneous activity or stable activity upon continuous small current flow $(<50 \mathrm{pA})$ were studied, and their AP characteristics analysed (see Section 2.2.4). This requirement dramatically reduced the number of cells eligible for experiments ${ }^{1}$. Pipette capacitance was compensated and the bridge balanced in all experiments before starting the recording. Voltages were recorded with an Axopatch 700B amplifier and digitised with a Digidata 1550A data acquisition system, using the pCLAMP 10.5 software (Molecular Devices). Pipettes were back-filled to avoid bubble formation in the tip and 1-2 $\mathrm{kPa}$ positive pressure was applied while approaching the cell to prevent tip contamination from cell debris. When in contact, negative pressure ( 2 to $5 \mathrm{kPa}$ ) was applied in order to promote gigaseal $(>1 \mathrm{G} \Omega$ ) formation. The voltage signal was sampled at $20 \mathrm{kHz}$ and low-pass filtered at 10

\footnotetext{
${ }^{1}$ Additional electrophysiological characterisation experiments through calcium imaging reported that only $\sim 30 \%$ of the F11s show spontaneous activity on their fifth differentiation day (data not shown).
}

$\mathrm{kHz}$. If the gigaseal was not formed in the first two minutes, cell and pipette were discarded.

\subsubsection{Combined mechanics and electrophysiology}

Patch stability under a $3 \mu \mathrm{m}$ loading was first confirmed in 3 cells via the "Membrane Test" option of Clampex (Molecular Devices) in voltage clamp mode, by evaluating the access resistance $R_{a}$ [23]. $R_{a}$ is required to vary by less than $20 \%$ during electrophysiological recordings to be considered stable. A protocol was established for which $R_{a}$ remained within the constrained values: gigaseals were $>1 \mathrm{G} \Omega$, the nanoindenter was kept as far as possible from the micropipette, the smallest possible nanoindenter step of $0.25 \mu \mathrm{m}$ was used when approaching the probe and the loading was carried out at small velocities $(3 \mu \mathrm{m} / \mathrm{s})$.

If a gigaseal was formed, and the cell showed ongoing spontaneous activity or stable activity under small positive current flow, the nanoindenter was then approached (see Figure 3) and the cell indented by $3 \mu \mathrm{m}$ for $80 \mathrm{~s}$, while tracking the cell's membrane potential time evolution in I0 mode; for more details, see Section 7. Nanoindenter and electrode signals were simultaneously recorded with a PicoScope 4262 (Pico Technology) sampled at $2 \mathrm{kHz}$. APs were detected and their features were analysed via custom MATLAB (MathWorks Inc.) codes.

\subsection{Numerical methods}

\subsubsection{Material properties calibration}

Taking as inputs nanoindentation force-displacement curves, custom MATLAB scripts were developed to determine the cell's LVR, fit the exponential decay of stressrelaxation experiments, extract the storage and loss moduli at each indentation frequency from DMA experiments and fit these data to a Prony series (details in Section 7).

\subsubsection{D FE simulation of the indentation}

A 3D FE simulation of the indentation was conducted to study the cell deformation. The cell 3D shape was acquired through a z-stack of live widefield images taken every $0.025 \mu \mathrm{m}$. Cells were incubated with CellMask Green Plasma membrane live stain (ThermoFisher) for $15 \mathrm{~min}$ at 1:1,000 dilution. The image was 3D-deconvoluted in AutoQuant $\mathrm{x} 3$ (Bitplane), the cell body cropped and its surface object created with Imaris 7.6.5 (Bitplane). The surface was then loaded into Autodesk Meshmixer software (Autodesk Inc.), meshed with 8,512 tetrahedral elements and finally exported to ABAQUS (Dassault Systèmes SE) to perform a FE simulation of the indentation using the material properties calibrated from DMA experiments. For implementation details, see Section 7.

\subsubsection{Membrane area driven electrophysiology alteration}

The cells' surface area change induced by the indentation was extracted from the deformed configuration of the FE simulation. This value was updated in a modified surfacedependent electrophysiological model of DRG cells [24] to 

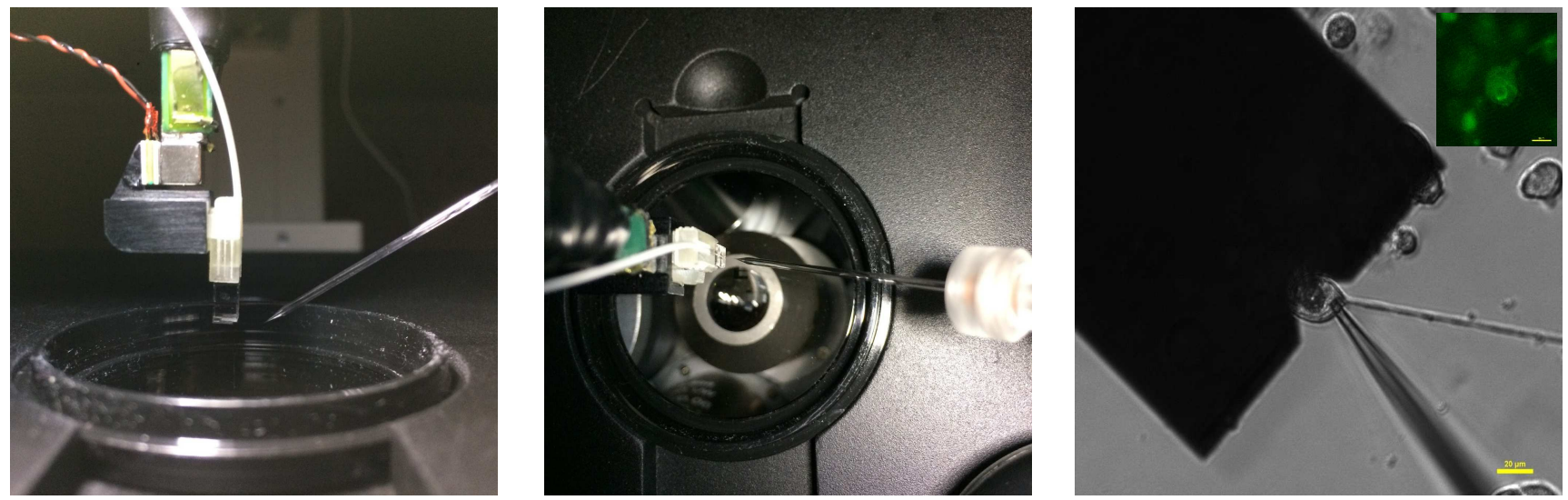

Figure 3: Left and centre: Nanoindenter and patch pipette arrangement. Right: both probes on the same F11 cell body. Inset: fluorescent image of a single cell with both probes. Scale bars are $20 \mu \mathrm{m}$.

study the effect of cell membrane area variation on membrane capacitance, when keeping constant the number of ion channels per unit reference area:

$$
\begin{aligned}
\frac{C_{m} A}{h_{m}} \frac{d V_{m}}{d t}=I_{i n j}- & \left(\frac{\bar{G}_{N a} A_{0}}{h_{m}} m^{3} h\left(V_{m}-E_{N a}\right)\right. \\
& +\frac{\bar{G}_{N a_{s}} A_{0}}{h_{m}} m_{s} h_{s}\left(V_{m}-E_{N a}\right) \\
& +\frac{\bar{G}_{K} A_{0}}{h_{m}} n\left(V_{m}-E_{K}\right) \\
& \left.+\frac{G_{L} A}{h_{m}}\left(V_{m}-E_{L}\right)\right)
\end{aligned}
$$

where $C_{m}$ is the membrane electric constant, $h_{m}$ is the membrane thickness, $V_{m}$ is the membrane potential, $A_{0}$ and $A$ are the undeformed and deformed total membrane areas, respectively, $I_{i n j}$ is the patch injected current, $\bar{G}_{N a}, \bar{G}_{N a_{s}}, \bar{G}_{K}$ and $G_{L}$ are the fast and slow-inactivated sodium, potassium and leak reference conductivities, $m, m_{s}, h, h_{s}$ and $n$ are the ion channels gating variables, and $E_{N a}, E_{K}$ and $E_{L}$ are the sodium, potassium and leak reference potentials. The main assumption behind this equation is that the area change does not alter the number of ion channels, but alters the membrane leak conductance and capacitance, as proposed in Ref. [25]. The values of the parameters and the evolution of the gating variables used in the simulation are those of a DRG cell [26] and are included in Tables 5, 6 and 7 of Section 7.

\subsubsection{AP features analysis}

The APs from the recorded voltage signal were detected and their features analysed via custom MATLAB codes. The experimental AP features were compared between the three phases of the indentation (before, during and after): AP amplitude, AP width, membrane resting voltage $V_{\text {res }}$, AP threshold voltage $V_{t h}$, rising and falling phases (see Figure 4), as well as AP firing frequency and maximum values of the first temporal derivative of the membrane potential, $\mathrm{d} V_{m} / \mathrm{dt}$. The AP amplitude is defined as the difference be-

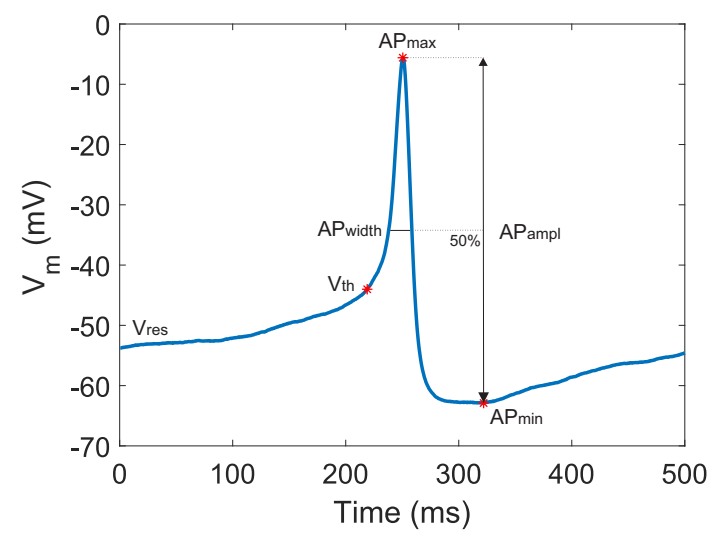

Figure 4: Analysed AP features.

tween the depolarisation peak and the maximum hyperpolarisation voltage values of the AP. The AP width is determined at half maximum AP amplitude. $V_{\text {res }}$ is defined as the value of the voltage $250 \mathrm{~ms}$ before the AP peak. $V_{t h}$ is defined as the voltage at which $\mathrm{d} V_{m} / \mathrm{dt}$ exceeds $0.35 \mathrm{mV} / \mathrm{ms}$ as determined by visual inspection of the reference membrane voltage $v s$. time curve. The rising phase is defined as the time lapse between the membrane potential at $V_{t h}$ to the peak of the AP, and the falling phase, the time lapse between the maximum and minimum values of the AP.

Although the cells under study were a priori selected based on the similarity of their phenotype, i.e, rounded cell bodies with neurites, the recorded APs had different cellspecific shapes and durations, even before indentation. This is however expected from the already mentioned multiple phenotypes found within F11s [20]. For comparison purposes, each averaged value of interest for each cell was normalised by dividing it by its counterpart before indentation. As voltage values during the APs remain always negative in the studied cells, both numbers are negative, the ratio is positive, and a normalised value $>1$ means that the cell hyperpolarised with respect to before the loading, while a nor- 
malised value $<1$ indicates a cell depolarisation during or after indentation. For temporal quantities, a ratio $>1$ illustrates a longer process, and $<1$ a shorter one. For reference, the original mean values are included in Appendix.

In the following, results are classified into reversible (or transient) and irreversible (or sustained). Reversible results are those for which the normalised mean values increase (or decrease) from before to during, but then decrease (or increase) back to the original values, see Figure 8, plotted together with the standard error (SE). If instead there are two consecutive increments or decrements, then the results are referred to as non-reversible, at least for the time-length of the study, see Figure 9.

\subsubsection{Statistical analysis}

The features of the APs were analysed via custom MATLAB codes. Statistical analyses of the post-processed electrophysiological data were performed with GraphPad Prism 6. The normality of data distribution was checked with Kolmogorov-Smirnov test, $\alpha=0.05$. In order to test the significance (defined with a threshold probability of 0.05 ) of the observed differences between the three experimental phases (before, during and after indentation) in terms of normalised mean values of the analysed AP features, oneway ANOVA with Tukey's multiple comparisons post-hoc tests were conducted if the data were normally distributed, and non-parametric Kruskal-Wallis test with Dunn's multiple comparisons post-hoc tests were carried out otherwise.

\section{Results}

\subsection{Mechanical characterisation of $F 11$ cells}

F11 cells' LVR was determined by fitting the Young's moduli of the subsequent 2, 3 and $4 \mu \mathrm{m}$ indentations (see details in Section 7), leading to values between 55 and $166 \mathrm{~Pa}$, see Figure 5. The results are in agreement with published values of Young's moduli for DRG and other neuronal cell types $[27,28]$. Although the values vary a lot (arising from the heterogeneity of phenotypes present within F11 populations [20]), each cell's Young's moduli at 3 and $4 \mu \mathrm{m}$ were within $10 \%$ of the value at $2 \mu \mathrm{m} ; 10 \%$ being the chosen criterion to ensure that these depths are within the LVR. A fixed indentation of $3 \mu \mathrm{m}$ was thus chosen for all coupled electrophysiological-mechanical experiments. Indentationcontrolled feedback loop ensured that this indentation depth was held constant throughout the experiment.

Single and double exponential fits of stress-relaxation experiments lead to calibrated relaxation time constants, $\tau_{1}$, of $2.77 \pm 0.67 \mathrm{~s}$ and $2.29 \pm 0.56 \mathrm{~s}$, respectively, see Appendix. In the double exponential case, only $\tau_{1}$ is physically relevant, as $\tau_{2}$ essentially captures the drifting of the system. As a compromise, $\tau_{1}=2.5 \mathrm{~s}$ was chosen for the numerical model as the time constant of the first branch (see Appendix for details).

For the DMA, the fitted mean and standard deviation (SD) storage and loss moduli values at $0.1,0.316,1,3.16$ and $10 \mathrm{~Hz}$ are given in Table 1 (see Section 7 for derivation details).

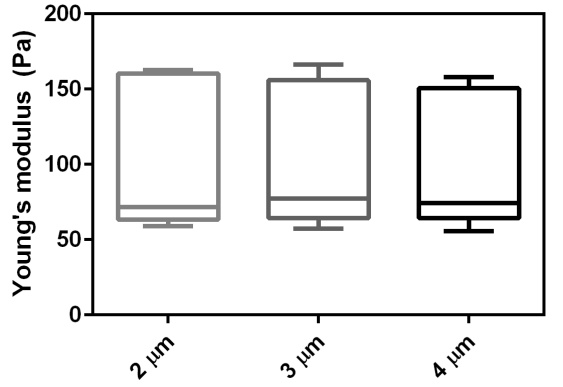

Figure 5: Boxplot showing the median, $\min$ and max fitted Young's moduli values for indentations of 2, 3 and $4 \mu \mathrm{m}$ depth in $\mathrm{N}=9$ cells.

\begin{tabular}{c||c|c|c} 
Freq $\mathbf{( H z )}$ & $\mathbf{E}^{\prime} \mathbf{( P a )}$ & $\mathbf{E}^{\prime \prime} \mathbf{( P a )}$ & $\tan \delta$ \\
\hline 0.1 & $105.63 \pm 32.09$ & $57.84 \pm 20.33$ & 0.55 \\
0.316 & $151.79 \pm 40.55$ & $86.71 \pm 28.62$ & 0.57 \\
1 & $210.29 \pm 59.62$ & $140.59 \pm 41.46$ & 0.67 \\
3.16 & $292.29 \pm 88.91$ & $250.43 \pm 70.43$ & 0.86 \\
10 & $421.36 \pm 61.86$ & $398.58 \pm 54.48$ & 0.95
\end{tabular}

Table 1

Storage (E') and loss (E') moduli mean and SD, and $\tan \delta$ values at each DMA frequency.

\subsection{AP alterations during and after indentation}

The features of a total of 284 spontaneous APs from $\mathrm{N}=5$ F11 cells were analysed and compared. The altered characteristics of the APs before, during and after mechanical loading for one of these cells are shown in Figure 6. To help the visualisation of those changes, an exagerated qualitative schematic of the AP shape and AP dynamics alterations induced by the quasi-static loading is shown in Figure 7. Comparisons were made for each cell in the three different indentation regimes: before, during and after (with a total of 115 APs before, 80 during and 89 after).

\subsubsection{Transient changes: Smaller, wider and slower rising APs upon indentation}

The normalised mean amplitudes of the APs are significantly reduced under loading (adjusted $* \mathrm{p}=0.0352$ ), recovering their original values after the $80 \mathrm{~s}$ indentation, see Figures 6-B and 8-A. APs during indentation are also significantly wider (adjusted $* p=0.0347$ ) and show the same recovery trend when the applied pressure is released (Figure 8-B). The widening of the peaks is an indication of slower dynamics of the electrical signals in cells under loading. To further investigate this, the rate of membrane voltage change over time $\left(\mathrm{d} V_{m} / \mathrm{dt}\right)$ and the duration of the rising AP phases were analysed. The maximum normalised mean $\mathrm{d} V_{m} / \mathrm{dt}$ value is significantly smaller under loading with respect to before (adjusted $* \mathrm{p}=0.0473$ ) and after (adjusted $* \mathrm{p}=0.0364$ ) indentation (Figure 8-C). Phase plots are obtained by plotting $\mathrm{d} V_{m} / \mathrm{dt}$ against $V_{m}$, see Figure 6-C which shows that the rate of voltage change is slower in a compressed cell (less pronounced depolarisation and repolari- 
A

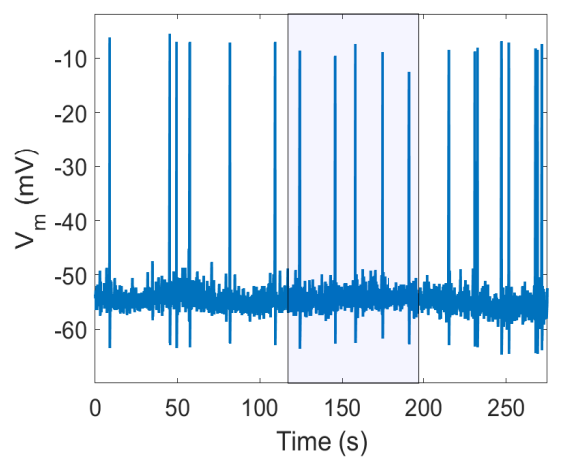

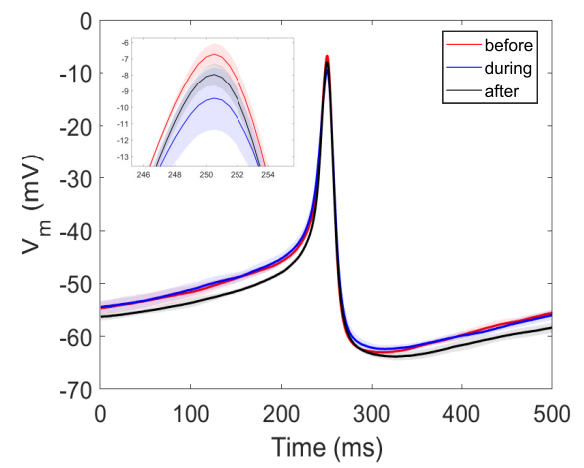

C

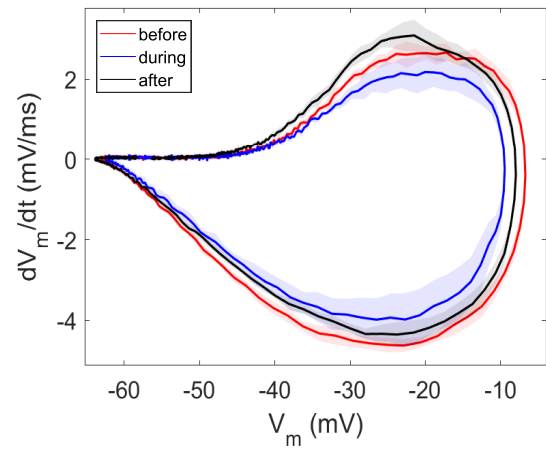

Figure 6: A) Spontaneous APs before, during (shaded) and after $3 \mu \mathrm{m}$ indentation for one of the analysed cells. B) Mean and SD of the APs on A), with peaks in the inset. C) Mean and SD of the phase plots $\left(\mathrm{d} V_{m} / \mathrm{dt} v s . V_{m}\right)$ for the APs on A).

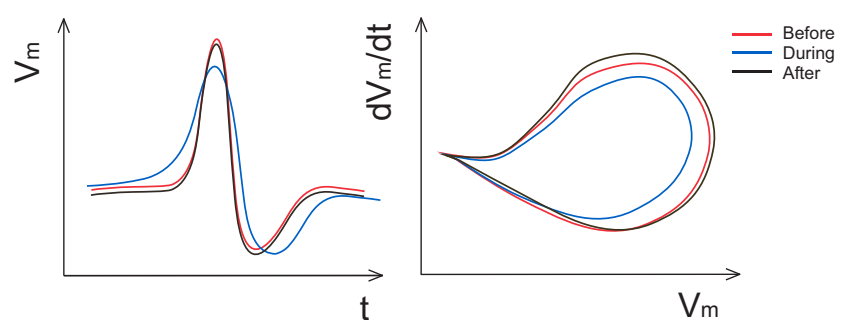

Figure 7: Qualitative representation of the AP shape and AP dynamics alterations induced by quasi-static loading of single F11 cell bodies. AP shape and evolution trends are exaggerated for better visibility.

sation slopes), with the altered dynamics recovering once the loading is removed. Figure 6-C further shows that APs of loaded cells reach smaller maximum values, as does Figure 8-A. In agreement with the mentioned slower dynamics, although non-significant, the rising phases of the APs are longer under loading and tend towards recovery after indentation (Figure 8-D), indicating that a compressed cell takes more time to depolarise (more gradual slope in Figure 6-C).

\subsubsection{Sustained changes: Hyperpolarised cells with longer repolarisations after indentation}

Non-reversible results show that cells remain hyperpolarised after indentation; at least for the few minutes recorded after the loading was halted. Normalised mean $V_{\text {res }}$ values are significantly hyperpolarised after indentation when compared to before and during (adjusted $* * p=0.0036$ and $* * \mathrm{p}=0.0065$, respectively, see Figure 9-A). The evolution of $V_{t h}$ reveals a similar result: normalised mean $V_{t h}$ values after indentation are significantly hyperpolarised with respect to before (adjusted $* * * p=0.0005$ ) and during (adjusted $* * * \mathrm{p}=0.0002$ ) loading. In addition, although not significant, mean $V_{t h}$ values during loading are slightly depolarised (see Figure 9-B), which will be discussed in Section 4. Despite cells being hyperpolarised during and after loading, on average they also fire more APs per s (Figure 9-C). Finally, even though AP widths tend to recover
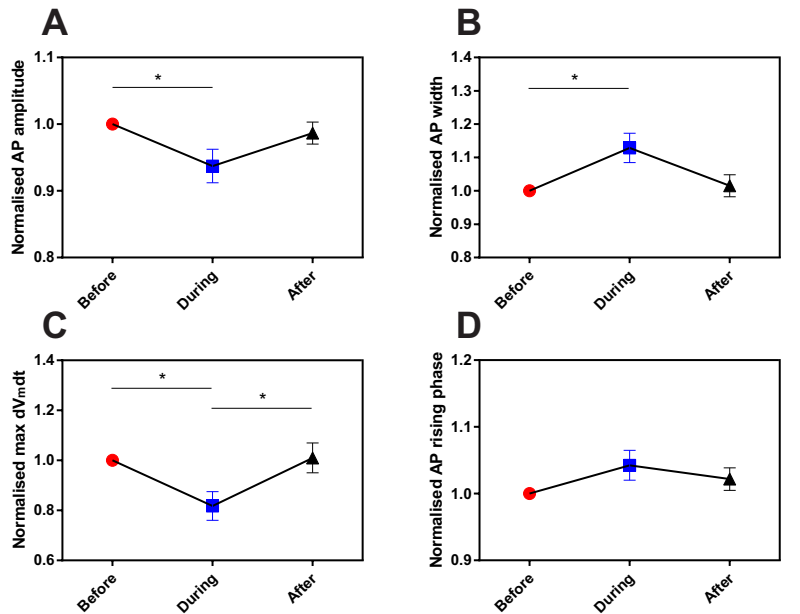

Figure 8: Mean and SE of the reversible or transient $A P$ changes. A) Normalised AP amplitudes. B) Normalised AP widths. C) Normalised max $\mathrm{d} V_{m} / \mathrm{dt}$ values. D) Normalised AP rising phases. In A), Kruskal-Wallis test was conducted with Dunn's multiple comparisons post-hoc test $\left({ }^{*} p<0.05\right)$. In B) and $($ ), one-way ANOVA was performed with a Tukey's multiple comparisons post-hoc test $\left({ }^{*} \mathrm{p}<0.05\right)$.

once the indentation is released (Figure 8-B), normalised mean falling phases are significantly longer after indentation $(* * \mathrm{p}=0.0075)$, see Figure 9-D. These longer repolarisation phases are in line with the more hyperpolarised voltage values the cell reaches after indentation.

\section{Discussion}

The normalised mean AP amplitudes during loading are significantly reduced when compared to those before indentation (Figures 6-B and 8-A). These values are reached more slowly, as shown by the significantly smaller maximum values of the $\mathrm{d} V_{m} / \mathrm{dt}$ evolution (Figures 6-C and 8-C) and longer rising phases (Figure 8-D) during loading. Furthermore, on average, cells take more time to repolarise back to their resting values during indentation, see Figure 9-D. Overall, this 

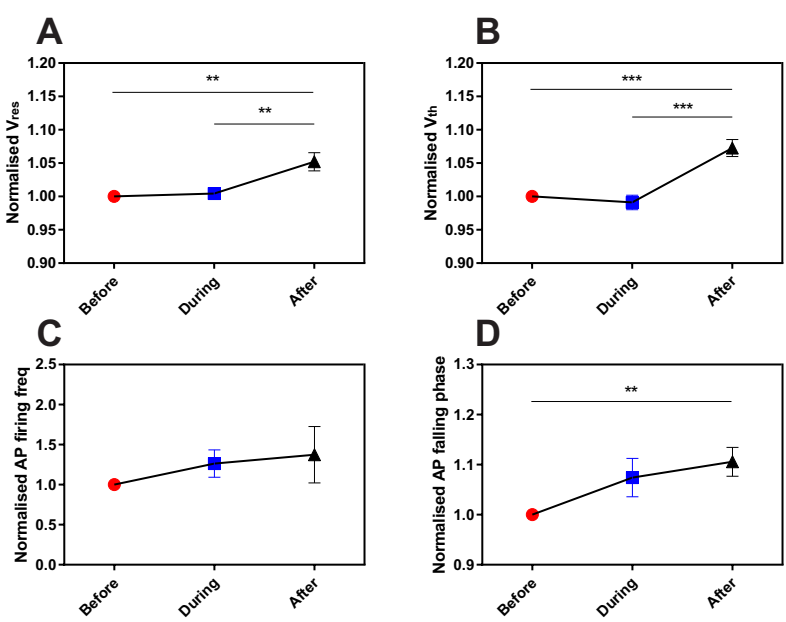

Figure 9: Mean and SE of the irreversible or sustained AP changes. A) Normalised resting membrane voltage values. B) Normalised threshold potential. C) Normalised AP firing frequencies. D) Normalised AP falling phases. In A) and B), one-way ANOVA was performed with a Tukey's multiple comparisons post-hoc test $\left({ }^{* *} \mathrm{p}<0.01 ;{ }^{* * *} \mathrm{p}<0.001\right)$. In D), Kruskal-Wallis test was performed, with Dunn's multiple comparisons post-hoc test $\left({ }^{* *} \mathrm{p}<0.01\right)$.

slower dynamics in turn results in a slower AP rate (Figure 6-C), or equivalently, wider APs (Figure 8-B), during loading.

Similar results have previously been found in experiments and numerical simulations. Experimentally, a decrease in AP amplitude was observed in guinea pig spinal cord compression [29] and in patients with suspected median and ulnar nerve lesions [30] or carpal tunnel syndrome [31]. In many of these cases, AP conduction velocity was also found to decrease with loading. Modelling efforts have led to similar results. For instance, in Ref. [32], the authors simulated the AP shape along a compressed axon, showing that its amplitude was reduced upon application of a force, and its conduction velocity decreased as the loading increased. Identical results were obtained when the AP propagation was modelled in axons subjected to stretch [33, 34].

In the following, we investigate the possible causes for these findings. As the indentations are within the cell's LVR, we assume that only membrane and membrane cortex are influenced by the loading. We first look at the more general contribution of the morphological nature of the membrane, accounting for the membrane geometry change and the corresponding induced voltage dynamics alteration. We then discuss the effects of the ion channels embedded in the membrane, focussing particularly on mechanosensitivity.

\subsection{Geometrical contribution}

An in silico FE neuronal soma was created from widefield microscopy, segmentation and meshing. A simulation of the cell indentation was performed in ABAQUS (see Figure 10 and Section 7 for details). The results showed an overall cell surface area increase of $0.4 \%$ for a $3 \mu \mathrm{m}$ indentation.

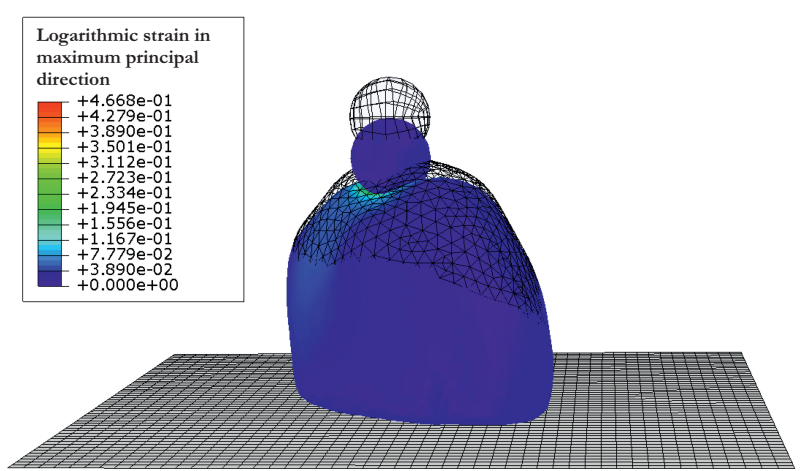

Figure 10: FE simulation of a F11 cell body indentation showing both the undeformed and deformed configurations; colour field corresponds to the logarithmic strain in the maximum principal direction.

This increase is in line with the assumption that the shape of a quasi-incompressible volume with surface tension (e.g., arising from the cell membrane) will naturally tend towards a sphere so as to minimise its surface energy, and thus area. As such, any mechanical disturbance is indeed expected to increase the area. Note that comparable 0.26 and $0.16 \%$ peak and steady-state membrane area changes arose in artificial protein-free lipid membranes stimulated with $1 \mathrm{MHz} \mathrm{US}$, as a result of the membrane displacement caused by the acoustic radiation force [35].

As Figure 10 shows, the higher values of the maximum principal strains in the compressed cell are localised at and around the area of direct contact with the glass bead. The three principal strain components of several elements, underneath the bead and in its direct neighbourhood, were also extracted in order to evaluate their external surface area change. Results showed that elements below the bead increased their external area by $\sim 40 \%$ when the cell was indented $3 \mu \mathrm{m}$, whereas those in the immediate vicinity of the nanoindenter experienced a $\sim 10 \%$ area increase. This indicates that heterogeneous and localised electrophysiological changes can be expected upon indentation (see Figure 15 in Section 7 for the effect of those area increments on the AP shape). However, these large alterations are significantly diluted (to $0.4 \%$ overall area change) when considering the entire cell membrane. It is worth mentioning that, although in this simulation the cell area increased, a less spherical cell body indented in a particular way could have seen instead its area decrease. While the results discussed here are thus cellspecific, the order of magnitude of the predicted changes is not expected to vary drastically. Similar curvature effects connecting cell geometry and depolarisation have also been discussed in Ref. [5].

The resolution of Equation (1) was implemented in MATLAB and the APs were simulated for the following two cell area values: $A_{0}$ (the non-indented cell area) and $A=1.004 A_{0}$. While the simulated APs followed the same trend observed experimentally, i.e., smaller and wider (or slower) APs (see Figure 11), the AP peak reduction only accounts for a fraction of the one observed experimentally 
A
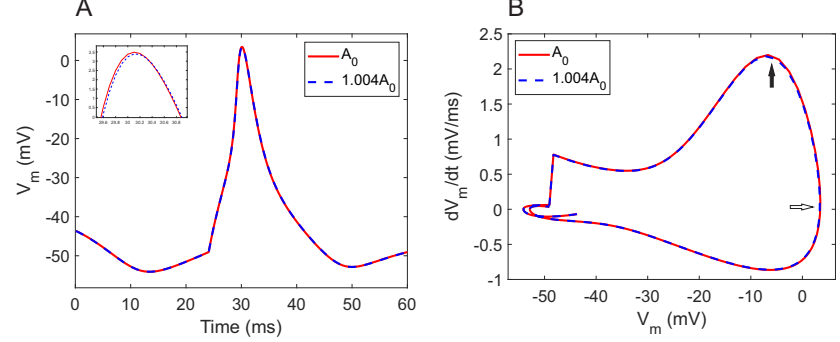

Figure 11: Modelled (through Equation (1)) DRG APs (A) and their phase-plots (B) before and during a $0.4 \%$ cell area increase, resulting from a $3 \mu \mathrm{m}$ cell indentation. Inset: AP peaks zoomed in. In B), unfilled and filled arrows point at smaller and slower APs, respectively, during loading (dashed blue curves).

(0.1 mV AP amplitude change, as opposed to the experimentally measured $\sim 2.53 \mathrm{mV}$ on average). In order to reach similar numerical values to those obtained experimentally in Figure 6, the overall cell area change had to be increased to $10 \%$, as Figure 15 in Section 7 shows. This discrepancy implies that other mechanisms are at play. In Equation (1), the membrane thickness $h_{m}$ was considered constant during deformation. Nonetheless, in the indented area and potentially other parts of the cell, the tension induced in the membrane by the indentation can potentially make it thinner through the Poisson effect, which would, similarly to a membrane area enlargement, increase the membrane capacitance $\left(C_{m}=\epsilon \frac{A}{h_{m}}\right)$, and slow down the voltage dynamics during an AP. Myelin wrapped around axons has the opposite effect of increasing the total insulating lipidic membrane thickness, which decreases the membrane capacitance and increases the AP propagation speed. Whether driven by area or thickness change, an overall cell membrane capacitance increase of $\sim 10 \%$ is thus required to recover the time-changing dynamics of Figure 15. Previous authors have shown that cell capacitance increase is a mechanism by which, through different means such as infrared light [36] and thermal transients [37], one can influence neuronal electrophysiology (in these cases, by depolarising the cells). While the effect of capacitance changes cannot be discarded without further investigations, both area and thickness (and thus capacitance) changes are predicted to be very small for most of the cell, and other mechanisms are most likely involved.

\subsection{Ion channels contribution}

Ion channels and their gating kinetics can be expected to be affected in a compressed membrane; especially those mechanically activated or mechanosensitive (MS). F11 cells are DRG hybrids, which are sensory neurons with MS traits. F11s respond with small currents to mechanical indentations [38] through endogenous mechano- and volumeactivated channels such as TRPV4 [39, 40] and Tentonin3 (TMEM150C) [38]. They also present neuronal markers of A- and C-fibre mechanosensors and proprioceptors, including LDHB, NEFH and TrkC [41]. Furthermore, ion channels with recognised roles in nociception such as
ASIC1, CaV3.2, KV7.2, TRPM3 [41] and bradykinin and prostaglandin receptors [18] are expressed in F11s.

Although not specifically on F11s, simultaneous indentation and electrophysiology recordings have been conducted in DRG cells to characterise indentation-evoked MS currents $[14,15,42]$. In particular, both rapid and slow adapting MS ion currents were recorded in DRGs under indentation [15]. These mechanically activated currents are non-selective cation inward currents that depolarise the cell membrane potential, in turn inactivating some sodium ion channels and contributing to smaller AP amplitudes, slower AP and longer rising phases, in line with what was observed for F11 quasi-static loading (Figure 8). Although cell depolarisation $\left(V_{\text {res }}\right)$ under loading was not observed in our experiments (Figure 9-A), normalised mean $V_{t h}$ values appear to be slightly reduced during loading (Figure 9-B), meaning that less depolarising current is needed to trigger an AP when the cell is being compressed.

After indentation, normalised mean $V_{\text {res }}$ values are significantly hyperpolarised (Figure 9-A) and the neuronal firing rate is increased (Figure 9-C). The repolarisation phase of the AP is mediated mainly by potassium currents, which also regulate AP firing frequency [43]. The longer AP falling phases observed after indentation (Figure 9-D) point towards activation of delayed rectifier potassium channels, which permit high frequency AP firing [44]. In addition, hyperexcitability has been observed in chronically compressed DRGs, as a result of an upregulation of hyperpolarisation-activated currents [45]. These are, similarly to MS currents, inward non-selective cation currents that flow through hyperpolarisation-activated cyclic nucleotide-gated channels, known to be present in DRG somas and axons. While these DRGs were compressed between five and seven days in order to induce a chronic response, it may be that hyperpolarisation-activated currents could arise in F11 cells in a much shorter time (here, after only $80 \mathrm{~s}$ indentation) and contribute to the observed irreversible enhanced hyperpolarisation and increased firing rates (Figure 9-A,C).

The observed irreversible (for the few minutes recorded after indentation) electrophysiological alterations could be the result of a compound ion channels' response, potentially also including nociceptive signals. However, studies have shown that even the slow adapting currents are only persistent for hundreds of milliseconds, with their amplitudes considerably decaying after $4 \mathrm{~s}$ [15]. As the observed AP shape changes are maintained throughout the $80 \mathrm{~s}$ indentation (see Figure 6-A), an exclusively ion channels' response is yet again unlikely to be the sole driver behind the observed sustained $80 \mathrm{~s}$ lasting changes.

\subsection{Multiphysics response}

As seen above, the sole contribution of either membrane topology or ion channel alterations is unlikely to account for the observed effects. A combined action, where membrane and ion channels (among other proteins) work concurrently and collaboratively through mechanical and bio- 
chemical changes, is proposed here instead. Such observation builds naturally on the growing body of evidence pointing towards the need to redefine the cellular membrane as a coupled multiphysics system [1]. In our experiments, the geometrical changes induced by the indentation affect the state of stress in the lipid bilayer, thus potentially altering the structure and function of the embedded ion channels $[46,47]$. MS channels and voltage-gated potassium [43, 4850] and sodium [51-53] channel gating kinetics are known to be affected by membrane tension and composition. In fact, previous work by the authors demonstrated that these same F11 cells showed smaller magnitude sodium currents when subjected to uniaxial strain [54]. The altered rising (Figure 8-D) and falling (Figure 9-D) phases during and after loading observed here could indicate that voltage-gated sodium and potassium channels kinetics, respectively, are altered by the applied mechanical load. Conversely, MS Piezo channels have been shown to be modulated by voltage [55]. These results, together with the data provided here, show that the mechanics of the neuronal membrane is intrinsically coupled to its electrophysiology.

The contribution of the cytoskeleton to the results introduced here is not believed to be very significant because of the indentation depth falling within the cell's LVR, thus $a$ priori pointing towards a limited biochemical reorganisation. A given degree of plastic deformation arising from bond ruptures within the cytoskeleton [56], in turn affecting the cell's electrophysiology [34] is still, however, possible. In Ref. [57], the authors argued that a negative (left-) shift found in the voltage-dependence of sodium channels was due to ruptures of sites of membrane attachment to cytoskeleton. Whether the irreversible AP characteristics observed here would recover over a longer period or are the product of some mechanical damage would need to be assessed in future work.

\section{Conclusion}

In this paper, we have presented a setup that facilitates single cell multiphysics study whereby electrophysiology and mechanics can be simultaneously controlled and measured. These initial results on the quasi-static indentation of F11 cells provide evidence of the mechanoelectrical coupling in neurons at the single cell level. In particular, altered APs in compressed neurons appear as the result of the interplay between membrane and ion channels topological and conformational alterations. In the proposed paradigm, all physical processes need to be accounted for simultaneously and collaboratively to capture the mechanics-driven functional alteration of neurons in the nervous system.

\section{Acknowledgements}

The authors acknowledge funding from the EPSRC Healthcare Technologies Challenge Award No. EP/N020987/1 and thank Prof. Stephen Tucker for the fruitful discussions on mechanosensitive ion channels in neurons.

\section{References}

[1] A. Jérusalem, Z. Al-Rekabi, H. Chen, A. Ercole, M. Malboubi, M. Tamayo-Elizalde, L. Verhagen, and S. Contera. Electrophysiological-mechanical coupling in the neuronal membrane and its role in ultrasound neuromodulation and general anaesthesia. Acta Biomaterialia, 97:116-140, 2019. ISSN 17427061. doi: 10.1016/j.actbio.2019.07.041. URL https://doi.org/10.1016/j.actbio. 2019.07.041.

[2] A. L. Hodgkin and A. F. Huxley. A Quantitative Description of Membrane Current and its Application to Conduction and Excitation in Nerves. The Journal of Physiology, 117:500-544, 1952. ISSN 00223751. doi: 10.1016/S0092-8240(05)80004-7.

[3] A. Gonzalez-Perez, L. D. Mosgaard, R. Budvytyte, E. VillagranVargas, A. D. Jackson, and T. Heimburg. Solitary electromechanical pulses in lobster neurons. Biophysical Chemistry, 216:51-59, 2016. ISSN 18734200. doi: 10.1016/j.bpc.2016.06.005.

[4] Y. Yang, X. W. Liu, H. Wang, H. Yu, Y. Guan, S. Wang, and N. Tao. Imaging Action Potential in Single Mammalian Neurons by Tracking the Accompanying Sub-Nanometer Mechanical Motion. American Chemical Society Nano, (5):4186-4193, 2018. ISSN 1936-0851. doi: 10.1021/acsnano.8b00867.

[5] T. Ling, K. C. Boyle, V. Zuckerman, T. Flores, C. Ramakrishnan, K. Deisseroth, and D.1 Palanker. High-speed interferometric imaging reveals dynamics of neuronal deformation during the action potential. Proceedings of the National Academy of Sciences of the United States of America, 117(19):10278-10285, 2020. ISSN 10916490. doi: 10. 1073/pnas.1920039117.

[6] S. Besch, K. V. Snyder, P. C. Zhang, and F. Sachs. Adapting the Quesant@ Nomad ${ }^{\mathrm{TM}}$ atomic force microscope for biology and patchclamp atomic force microscopy. Cell Biochemistry and Biophysics, 39(3):195-210, 2003. ISSN 10859195. doi: 10.1385/CBB:39:3:195.

[7] J. Mosbacher, M. Langer, J. K. Hörber, and F. Sachs. Voltagedependent membrane displacements measured by atomic force microscopy. The Journal of General Physiology, 111(1):65-74, 1998.

[8] A. Beyder and F. Sachs. Electromechanical coupling in the membranes of Shaker-transfected HEK cells. Proceedings of the National Academy of Sciences of the United States of America, 106(16):66266631, 2009. ISSN 00278424. doi: 10.1073/pnas.0808045106.

[9] E. Pamir, N. George, M.and Fertig, and M. Benoit. Planar patchclamp force microscopy on living cells. Ultramicroscopy, 108(6): 552-557, 2008. ISSN 03043991. doi: 10.1016/j.ultramic.2007.08. 013.

[10] K. V. Upadhye, J. E. Candiello, L. A. Davidson, and H. Lin. Wholecell electrical activity under direct mechanical stimulus by AFM cantilever using planar patch clamp chip approach. Cellular and Molecular Bioengineering, 4(2):270-280, 2011. ISSN 18655025. doi: 10.1007/s12195-011-0160-4.

[11] C. Zhang, P. Li, L. Liu, Y. Wang, Z. Gao, and G. Li. Development of mechanostimulated patch-clamp system for cellular physiological study. IEEE/ASME Transactions on Mechatronics, 19(4):1138-1147, 2014. ISSN 10834435. doi: 10.1109/TMECH.2013.2272562.

[12] D. Ossola, L. Dorwling-Carter, H. Dermutz, P. Behr, J. Vörös, and T. Zambelli. Simultaneous Scanning Ion Conductance Microscopy and Atomic Force Microscopy with Microchanneled Cantilevers. Physical Review Letters, 115(23):1-5, 2015. ISSN 10797114. doi: 10.1103/PhysRevLett.115.238103.

[13] B. Coste, J. Mathur, M. Schmidt, T. J Earley, S. Ranade, M. J. Petrus, A. E. Dubin, and A. Patapoutian. Piezo1 and Piezo2 Are Essential Components of Distinct Mechanically Activated Cation Channels. Science (New York, N.Y.), 330(October):7-12, 2010. ISSN 00368075. doi: 10.1126/science. 1193270.

[14] J. Hao and P. Delmas. Recording of mechanosensitive currents using piezoelectrically driven mechanostimulator. Nature protocols, 6(7): 979-990, 2011. ISSN 1754-2189. doi: 10.1038/nprot.2011.343. URL http://dx.doi.org/10.1038/nprot. 2011.343.

[15] F. Rugiero, L. J. Drew, and J. N. Wood. Kinetic properties of mechanically activated currents in spinal sensory neurons. The Journal of Physiology, 588(2):301-314, 2010. ISSN 00223751. doi: 
10.1113/jphysiol.2009.182360.

[16] S. Sianati, A. Kurumlian, E. Bailey, and K. Poole. Analysis of mechanically activated ion channels at the cell-substrate interface: Combining pillar arrays and whole-cell patch-clamp. Frontiers in Bioengineering and Biotechnology, 7(MAR):1-12, 2019. ISSN 22964185. doi: 10.3389/fbioe.2019.00047.

[17] A. L. Eastwood, A. Sanzeni, B. C. Petzold, S. J. Park, M. Vergassola, B. L. Pruitt, and M. B. Goodman. Tissue mechanics govern the rapidly adapting and symmetrical response to touch. Proceedings of the National Academy of Sciences of the United States of America, 113(17): E2471, 2016. ISSN 10916490. doi: 10.1073/pnas.1604954113.

[18] P. C. Francel, K. Harris, M. Smith, M. C. Fishman, G. Dawson, and R. J. Miller. Neurochemical Characteristics of a Novel Dorsal Root Ganglion X Neuroblastoma Hybrid Cell Line, F-11. Journal of Neurochemistry, 48(5):1624-1631, 1987.

[19] D. Platika, M. H. Boulos, L. Baizer, and M. C. Fishman. Neuronal traits of clonal cell lines derived by fusion of dorsal root ganglia neurons with neuroblastoma cells. Proceedings of the National Academy of Sciences of the United States of America, 82(May):3499-3503, 1985.

[20] L. M. Boland and R. Dingledine. Expression of sensory neuron antigens by a dorsal root ganglion cell line, F-11. Developmental Brain Research, 51(2):259-266, 1990. URL http://www. ncbi.nlm.nih.gov/ pubmed/1969775.

[21] Y. T. Wu and A. Adnan. Damage and Failure of Axonal Microtubule under Extreme High Strain Rate: An In-Silico Molecular Dynamics Study. Scientific Reports, 8(1):1-9, 2018. ISSN 20452322. doi: 10.1038/s41598-018-29804-w. URL http://dx.doi.org/10. 1038/s41598-018-29804-w.

[22] M. Malboubi, Y. Gu, and K. Jiang. Characterization of surface properties of glass micropipettes using SEM stereoscopic technique. Microelectronic Engineering, 88(8):2666-2670, 2011. ISSN 01679317. doi: 10.1016/j.mee.2011.02.029.

[23] C. C. H. Petersen. Whole-Cell Recording of Neuronal Membrane Potential during Behavior. Neuron, 95(6):1266-1281, 2017. ISSN 10974199. doi: 10.1016/j.neuron.2017.06.049. URL https://doi. org/10.1016/j. neuron. 2017.06.049.

[24] H. Chen, D. Garcia-Gonzalez, and A. Jérusalem. Computational model of the mechanoelectrophysiological coupling in axons with application to neuromodulation. Physical Review E, 99(3):1-18, 2019. ISSN 24700053. doi: 10.1103/PhysRevE.99.032406.

[25] M. T. Kwong, F. Bianchi, M. Malboubi, J. A. García-Grajales, L. Homsi, M. Thompson, H. Ye, L. Noels, and A. Jérusalem. 3D finite element formulation for mechanical-electrophysiological coupling in axonopathy. Computer Methods in Applied Mechanics and Engineering, 346:1025-1050, 2019. ISSN 00457825. doi: 10.1016/ j.cma.2018.09.006.

[26] R. I. Herzog, T. R. Cummins, and S. G. Waxman. Persistent TTXresistant $\mathrm{Na}$ + current affects resting potential and response to depolarization in simulated spinal sensory neurons. Journal of Neurophysiology, 86(3):1351-1364, 2001. ISSN 00223077.

[27] E. Spedden, J. D. White, E. N. Naumova, D. L. Kaplan, and C. Staii. Elasticity maps of living neurons measured by combined fluorescence and atomic force microscopy. Biophysical Journal, 103(5):868-877, 2012. ISSN 00063495. doi: 10.1016/j.bpj.2012.08.005.

[28] Y. B. Lu, K. Franze, G. Seifert, C. Steinhäuser, F. Kirchhoff, H. Wolburg, J. Guck, P. Janmey, E. Q. Wei, J. Käs, and A. Reichenbach. Viscoelastic properties of individual glial cells and neurons in the CNS. Proceedings of the National Academy of Sciences, 103(47): 17759-17764, 2006. ISSN 0027-8424, 1091-6490. doi: 10.1073/ pnas.0606150103. URL http://www. pnas. org/content/103/47/17759. full. pdf.

[29] R. Shi and A.R. Blight. Compression injury of mammalian spinal cord in vitro and the dynamics of action potential conduction failure. Journal of Neurophysiology, 76(3):1572-1580, 1996. ISSN 00223077. doi: 10.1152/jn.1996.76.3.1572.

[30] R. W. Gilliatt and T. Sears. Sensory nerve action potentials in patients with peripheral lesions. Journal of Neurology, Neurosurgery and Psychiatry, 83(12):1137-1138, 1958. ISSN 1468330X. doi: 10.1136/jnnp-2012-303569.

[31] B. V. Watson, W. F. Brown, and T. J. Doherty. Frequency-dependent conduction block in carpal Tunnel syndrome. Muscle and Nerve, 33 (5):619-626, 2006. ISSN 0148639X. doi: 10.1002/mus.20513.

[32] T. Tekieh, S. Shahzadi, H. Rafii-Tabar, and P. Sasanpour. Are deformed neurons electrophysiologically altered? A simulation study. Current Applied Physics, 16(10):1413-1417, 2016. ISSN 15671739. doi: 10.1016/j.cap.2016.07.012.

[33] J. A. Garcia-Grajales, G. Rucabado, A. Garcia-Dopico, J. M. Pena, and A. Jérusalem. Neurite, a finite difference large scale parallel program for the simulation of electrical signal propagation in neurites under mechanical loading. Public Library of Science ONE, 10(2): 1-22, 2015. ISSN 19326203. doi: 10.1371/journal.pone.0116532.

[34] J. Tian, G. Huang, M. Lin, J. Qiu, B. Sha, T. J. Lu, and F. Xu. A mechanoelectrical coupling model of neurons under stretching. Journal of the Mechanical Behavior of Biomedical Materials, 93(February): 213-221, 2019. ISSN 18780180. doi: 10.1016/j.jmbbm.2019.02.007. URL https://doi .org/10.1016/j.jmbbm. 2019.02.007.

[35] M. L. Prieto, O. Oralkan, B. T. Khuri-Yakub, and M. C. Maduke. Dynamic Response of Model Lipid Membranes to Ultrasonic Radiation Force. Public Library of Science ONE, 8(10), 2013. ISSN 19326203. doi: 10.1371/journal.pone.0077115.

[36] M. G Shapiro, K. Homma, S. Villarreal, C. P. Richter, and F. Bezanilla. Infrared light excites cells by changing their electrical capacitance. Nature Communications, 3(April 2017):736, 2012. ISSN 2041-1723. doi: 10.1038/ncomms1742. URL http://www. nature. com/doifinder/10.1038/ncomms 1742 .

[37] M. Plaksin, E. Shapira, E. Kimmel, and S. Shoham. Thermal Transients Excite Neurons through Universal Intramembrane Mechanoelectrical Effects. Physical Review X, 8(1):11043, 2018. ISSN 21603308. doi: 10.1103/PhysRevX.8.011043. URL https://doi. org/10.1103/PhysRevX.8.011043.

[38] G. S. Hong, B. Lee, J. Wee, H. Chun, H. Kim, J. Jung, J. Yo. Cha, T. R. Riew, G. H. Kim, I. B. Kim, and U. Oh. Tentonin 3/TMEM150c Confers Distinct Mechanosensitive Currents in Dorsal-Root Ganglion Neurons with Proprioceptive Function. Neuron, 91(1):107-118, 2016. ISSN 10974199. doi: 10.1016/j.neuron.2016.05.029. URL http://dx.doi.org/10.1016/j. neuron. 2016.05.029.

[39] F. L. P. Bender, M. Mederos Y Schnitzler, Y. Li, A. Ji, E. Weihe, T. Gudermann, and M. K. H. Schäfer. The temperature-sensitive ion channel TRPV2 is endogenously expressed and functional in the primary sensory cell line F-11. Cellular Physiology and Biochemistry, 15(1-4):183-194, 2005.

[40] C. Goswami, J. Kuhn, P. A. Heppenstall, and T. Hucho. Importance of non-selective cation channel TRPV4 interaction with cytoskeleton and their reciprocal regulations in cultured cells. Public Library of Science ONE, 5(7):19-21, 2010. ISSN 19326203. doi: 10.1371/ journal.pone.0011654.

[41] K. Yin, G. J. Baillie, and I. Vetter. Neuronal cell lines as model dorsal root ganglion neurons. Molecular Pain, 12:174480691664611, 2016. ISSN 1744-8069. doi: 10.1177/1744806916646111. URL http:// journals. sagepub.com/doi/10.1177/1744806916646111.

[42] D. Usoskin, M. Zilberter, S. Linnarsson, J. Hjerling-Leffler, P. Uhlén, T. Harkany, and P. Ernfors. En masse in vitro functional profiling of the axonal mechanosensitivity of sensory neurons. Proceedings of the National Academy of Sciences of the United States of America, 107(37):16336-41, 2010. ISSN 1091-6490. doi: 10.1073/pnas. 0914705107.

[43] D. Oliver, C. C. Lien, M. Soom, T. Baukrowitz, P. Jonas, and B. Fakler. Functional Conversion between A-Type and Delayed Rectifier K+ Channels by Membrane Lipids. Science, 304(5668):265-270, 2004. ISSN 00368075. doi: 10.1126/science.1094113.

[44] C. C. Lien and P. Jonas. Kv3 potassium conductance is necessary and kinetically optimized for high-frequency action potential generation in hippocampal interneurons. Journal of Neuroscience, 23(6):20582068, 2003. ISSN 02706474. doi: 10.1523/jneurosci.23-06-02058. 2003. 
[45] H. Yao, D. F. Donnelly, C. Ma, and R. H. LaMotte. Upregulation of the hyperpolarization-activated cation current after chronic compression of the dorsal root ganglion. Journal of Neuroscience, 23 (6):2069-2074, 2003. ISSN 02706474. doi: 10.1523/jneurosci. 23-06-02069.2003.

[46] R. Phillips, T. Ursell, P. Wiggins, and P. Sens. Emerging roles for lipids in shaping membrane-protein function. Nature, 459(7245): 379-385, 2009.

[47] W. J. Tyler. The mechanobiology of brain function. Nature Reviews Neuroscience, 13(12):867-878, 2012.

[48] D. Schmidt and R. MacKinnon. Voltage-dependent K+ channel gating and voltage sensor toxin sensitivity depend on the mechanical state of the lipid membrane. Proceedings of the National Academy of Sciences of the United States of America, 105(49):19276-19281, 2008. ISSN 00278424. doi: 10.1073/pnas.0810187105.

[49] S. S. Ranade, R. Syeda, and A. Patapoutian. Mechanically Activated Ion Channels. Neuron, 87(6):1162-1179, 2015. ISSN 10974199. doi: 10.1016/j.neuron.2015.08.032. URL http://dx.doi.org/10.1016/j. neuron. 2015.08.032

[50] C. E. Morris, E. A. Prikryl, and B. Joós. Mechanosensitive gating of Kv channels. Public Library of Science ONE, 10(2):1-24, 2015. ISSN 19326203. doi: 10.1371/journal.pone.0118335.

[51] C. E. Morris and P. F. Juranka. Nav channel mechanosensitivity: activation and inactivation accelerate reversibly with stretch. Biophysical journal, 93(3):822-833, 2007.

[52] J. A. Wang, W. Lin, T. Morris, U. Banderali, P. F Juranka, and C. E. Morris. Membrane trauma and $\mathrm{Na}+$ leak from Nav1 . 6 channels Membrane trauma and Na leak from Nav1 . 6 channels. American Journal of Physiology-Cell Physiology, 297:823-834, 2009. doi: 10. 1152/ajpcell.00505.2008.

[53] L. J. Drew. Sodium channel mechanosensitivity: Pay a-tension to voltage sensor movement. Journal of Physiology, 589(5):1003-1004, 2011. ISSN 00223751. doi: 10.1113/jphysiol.2011.205880.

[54] F. Bianchi, M. Malboubi, J. H. George, A. Jérusalem, M. S. Thompson, and $\mathrm{H}$. Ye. Ion current and action potential alterations in peripheral neurons subject to uniaxial strain. Journal of Neuroscience Research, 97(7):744-751, 2019. ISSN 10974547. doi: 10.1002/jnr. 24408.

[55] M. Moroni, M. R. Servin-Vences, R. Fleischer, O. Sánchez-Carranza, and G.R. Lewin. Voltage-gating of mechanosensitive PIEZO channels. Nature Communications, 9(1096), 2018. ISSN 2041-1723. doi: 10.1101/156489.

[56] N. Bonakdar, R. Gerum, M. Kuhn, M. Spörrer, A. Lippert, W. Schneider, K. E. Aifantis, and B. Fabry. Mechanical plasticity of cells. Nature Materials, 15(10):1090-1094, 2016. ISSN 14764660. doi: 10.1038/nmat4689.

[57] A. Shcherbatko, F. Ono, G. Mandel, and P. Brehm. Voltage-dependent sodium channel function is regulated through membrane mechanics. Biophysical Journal, 77(4):1945-1959, 1999. ISSN 00063495. doi: 10.1016/S0006-3495(99)77036-0. URL http://dx.doi.org/10.1016/ s0006-3495(99)77036-0.

[58] S. Moreno-Flores, R. Benitez, M. d.M. Vivanco, and J. L. TocaHerrera. Stress relaxation microscopy: Imaging local stress in cells. Journal of Biomechanics, 43(2):349-354, 2010. ISSN 00219290. doi: 10.1016/j.jbiomech.2009.07.037.

[59] A. Bonfanti, J. Fouchard, N. Khalilgharibi, G. Charras, and A. Kabla. A unified rheological model for cells and cellularised materials. Royal Society Open Science, 7(1), 2020. ISSN 20545703. doi: 10.1098/rsos. 190920.

[60] K. L. Johnson. Contact mechanics. Cambridge University Press (\{CUP\}), 1985

[61] L. Bartolini, D. Iannuzzi, and G. Mattei. Comparison of frequency and strain-rate domain mechanical characterization. Scientific Reports, 8(1):1-11, 2018. ISSN 20452322. doi: 10.1038/s41598-018-31737-3. URL http://dx.doi.org/10.1038/ s41598-018-31737-3.

\section{Appendix}

\subsection{F11 expansion and differentiation media}

F11s were expanded in high glucose DMEM (ThermoFisher) with $10 \%$ fetal bovine serum (FBS) (ThermoFisher) and $1 \%$ penicillin/streptomycin (P/S) (ThermoFisher) in a humidified incubator at $37^{\circ} \mathrm{C}$ and $5 \% \mathrm{CO}_{2}$, changing media every three days. For differentiation, $\sim 5 \times 10^{4}$ cells were seeded per glass bottom dish (WillCoWells), in high glucose DMEM, supplemented with $1 \%$ FBS, $1 \% \mathrm{P} / \mathrm{S}, 0.5 \%$ insulin transferrin selenium (ThermoFisher), $10 \mu \mathrm{M}$ 3-isobutyl-1-methylx- anthine (Sigma-Aldrich), 50 $\mathrm{ng} / \mathrm{mL}$ nerve growth factor (Peprotech), $2 \mu \mathrm{M}$ retinoic acid (Sigma-Aldrich) and $0.5 \mathrm{mM}$ bromoadenosine 3,5-cyclic monophosphate, following Ref. [20]. Cells were differentiated for five days [18] prior to experiments, topping up the media every two days.

\subsection{Intra- and extracellular solutions}

The intracellular or pipette solution contained $140 \mathrm{mM}$ $\mathrm{KCl}, 5 \mathrm{mM} \mathrm{NaCl}, 0.1 \mathrm{mM} \mathrm{CaCl} 2,2 \mathrm{mM} \mathrm{MgCl} 2,10 \mathrm{mM} 4-$ (2-hydroxyethyl)-1-piperazineethanesulfonic acid (HEPES), $1 \mathrm{mM}$ guanosine-5'-triphosphate sodium salt hydrate, 2 $\mathrm{mM}$ adenosine triphosphate magnesium salt hydrate (mgATP) and $1 \mathrm{mM}$ ethylene glycol-bis( $\beta$-aminoethyl ether)$\mathrm{N}, \mathrm{N}, \mathrm{N}^{\prime}, \mathrm{N}^{\prime}$-tetraacetic acid (EGTA), with $\mathrm{pH}$ adjusted to 7.3 by addition of $\mathrm{KOH}$ and osmolarity adjusted to $290 \mathrm{mOsm} / \mathrm{L}$ by glucose addition. The intracellular solution was aliquoted into $500 \mu \mathrm{L}$, stored at $-20^{\circ} \mathrm{C}$ and filtered through a $0.22 \mu \mathrm{m}$ filter after thawing, on the day of experiments. The extracellular or bath solution contained $130 \mathrm{mM} \mathrm{NaCl}, 5 \mathrm{mM} \mathrm{KCl}, 2$ $\mathrm{mM} \mathrm{CaCl} 2,1 \mathrm{mM} \mathrm{MgCl} 2,10 \mathrm{mM}$ glucose, $10 \mathrm{mM}$ HEPES, with $\mathrm{pH}$ adjusted to 7.4 by addition of $\mathrm{NaOH}$ and osmolarity adjusted to $300 \mathrm{mOsm} / \mathrm{L}$ by glucose addition. The bath solution was filtered through a $0.22 \mu \mathrm{m}$ filter, aliquoted into $5 \mathrm{~mL}$ and stored at $4^{\circ} \mathrm{C}$. All reactants were purchased from Sigma Aldrich.

\subsection{Combined electrophysiology and nanoindentation}

After calibrating the nanoindenter, $2 \mathrm{~mL}$ of warmed bath solution were added to a glass bottom dish of F11 differentiated cells. Microelectrode and nanoindenter approached the cell from opposite $\left(180^{\circ}\right)$ sides (see Figure 3 left and middle, and Figure 12). In order to minimise seal instability and the pipette influencing the mechanical measurement, the cell was patched as close to the edge as possible. Once a good and stable seal of $>1 \mathrm{G} \Omega$ was obtained, the patched membrane was broken by gentle suction. In some cases, the "zap" option was used to promote membrane breakage, although it was found that membranes broken by zapping had a higher probability of membrane reseal. In whole-cell configuration and I0 mode, after two minutes of stabilisation (dialysis) of intracellular solution and cytoplasm, and pipette capacitance and bridge compensations, the recording of spontaneous or induced stable activity was started. After one minute of activity recorded before the indentation, the nanoindenter was manually positioned above the patched cell, opposite 


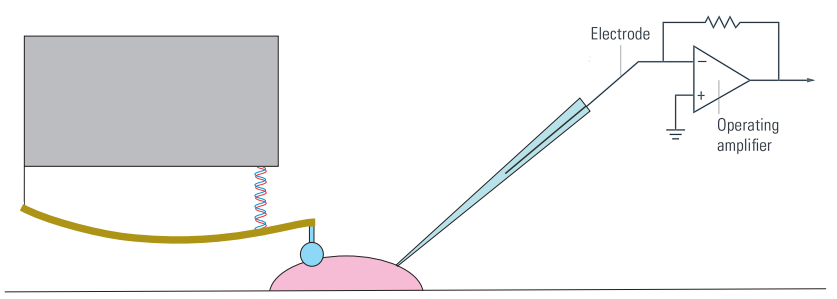

Figure 12: Schematic of the combined nanoindentation and patch clamp techniques on a single cell.

\begin{tabular}{c||c|c} 
Fit type & $\tau_{\mathbf{1}} \mathbf{( s )}$ & $\tau_{\mathbf{2}}(\mathbf{s})$ \\
\hline Single exponential decay & $2.77 \pm 0.67$ & \\
Double exponential decay & $2.29 \pm 0.56$ & $103.68 \pm 94.98$
\end{tabular}

Table 2

Mean and SD time relaxation values obtained by fitting stressrelaxation curves with one and two exponential decays.

the microelectrode (see Figure 3 right). The nanoindenter was manually brought into contact with the cell by $0.25 \mu \mathrm{m}$ z-steps and then retracted 1-2 $\mu \mathrm{m}$ above the cell surface. These small movements did not displace the micropipette, whose position was continuously monitored through brightfield imaging. A $3 \mu \mathrm{m}$ indentation-controlled loading was then started and held for $80 \mathrm{~s}$, and the activity recorded $d u r$ ing loading, after which the probe was withdrawn a few tens of $\mu \mathrm{m}$ above the cell and the activity further recorded (after loading) for another minute. Nanoindenter and cell membrane voltage signals were simultaneously recorded with a PicoScope 4262 (Pico Technology), sampled at $2 \mathrm{kHz}$. APs were detected and their features analysed via custom MATLAB codes.

\subsection{Stress relaxation fitting}

As a first approximation, a single exponential decay was used to fit the stress-relaxation curve via nonlinear least squares regression, by fixing the peak and plateau, see Figure 13. In order to better capture the initial decay, however, a double-exponential is commonly used $[58,59]$, of the form:

$$
F=F_{\infty}+a_{1} \exp \left(-t / \tau_{1}\right)+a_{2} \exp \left(-t / \tau_{2}\right)
$$

with $F_{\infty}$ is the load value at equilibrium, when the cell has fully relaxed, and $\tau_{1}$ and $\tau_{2}$ are the characteristic time responses of the material, corresponding to viscoelastic relaxation mechanisms.

Results of the single exponential fit were compared to the double exponential fit (MATLAB's “exp2”) by only fixing the plateau, see Figure 13. Mean and SD values of time relaxation values are shown in Table 2.

\subsection{LVR determination}

F11 cells can be considered, as a first approximation, as nonlinear viscoelastic: their mechanical response varies

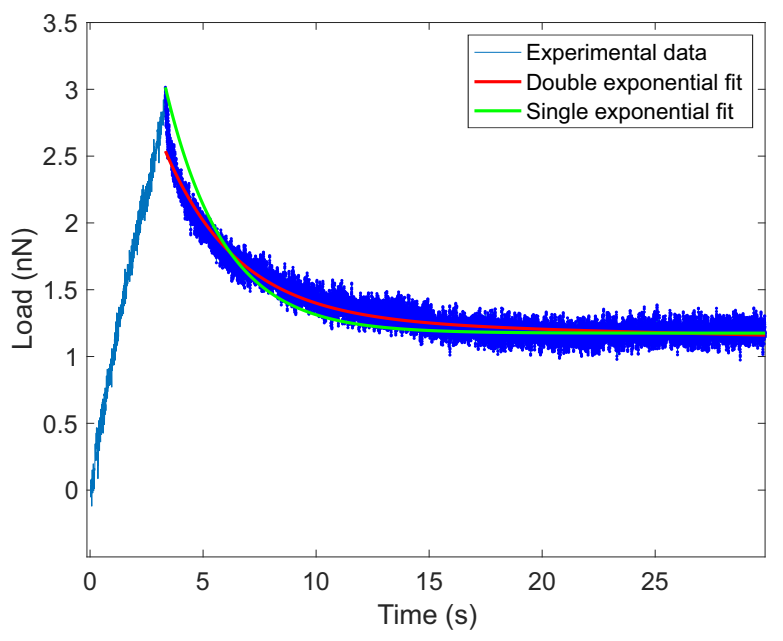

Figure 13: Single (in green) and double (in red) exponential decay fit of a stress-relaxation experiment from of a $2 \mu \mathrm{m} \mathrm{F11}$ cell indentation for $30 \mathrm{~s}$.

with indentation depth, due to the different mechanical natures of the membrane, membrane cortex, cytoplasm, organelles and nucleus. However, for small enough strains, the cell viscoelastic response is linear and as a result, its stiffness does not vary significantly (and is thus a "Young's modulus"). The LVR of a material under strain is defined as that in which the Young's modulus can be calculated within a $10 \%$ variance.

To identify the F11s' LVR, cells were indented at increasing depths $(2,3$ and $4 \mu \mathrm{m})$, giving them enough time (20 s) to relax after each indentation. For each case, the loading part of the force-indentation curve was fitted to the Hertz model. The Hertz model assumes that, for small enough deformations, an isotropic material behaves as linear elastic, and the material responds with a force $F$ to an indentation depth $h$ following [60]:

$$
F=\frac{4}{3} \frac{E}{\left(1-v^{2}\right)} R^{1 / 2} h^{3 / 2}
$$

where $E$ is the Young's modulus, $v$ is the Poisson's ratio (assume that cells are incompressible, $v \sim 0.5$ ) and $R$ is the indenter's spherical tip radius.

The loading region of the force-indentation curves was fitted via nonlinear least squares regression (fit type $F=$ $a \times h^{3 / 2}+F_{0}$, with $F_{0}$ being the value of the load at the point of contact) with a custom MATLAB code. The point of contact was manually estimated from the curves. The obtained Young's moduli might be overestimated because of adhesion forces, but as this systematic error is present in all the samples and indentations, their direct comparison should not $a$ priori be affected. A boxplot of the Young's moduli at indentations of 2, 3 and $4 \mu \mathrm{m}$ for $\mathrm{N}=9$ cells is shown in Figure 5. The Young's modulus of F11 cells at small indentations is between 55 and $166 \mathrm{~Pa}$, in agreement with previous results on DRG elastic moduli values and other neuronal cell types $[27,28]$. The variability on the measured Young's moduli comes from the different phenotypes present in F11s [20]. 


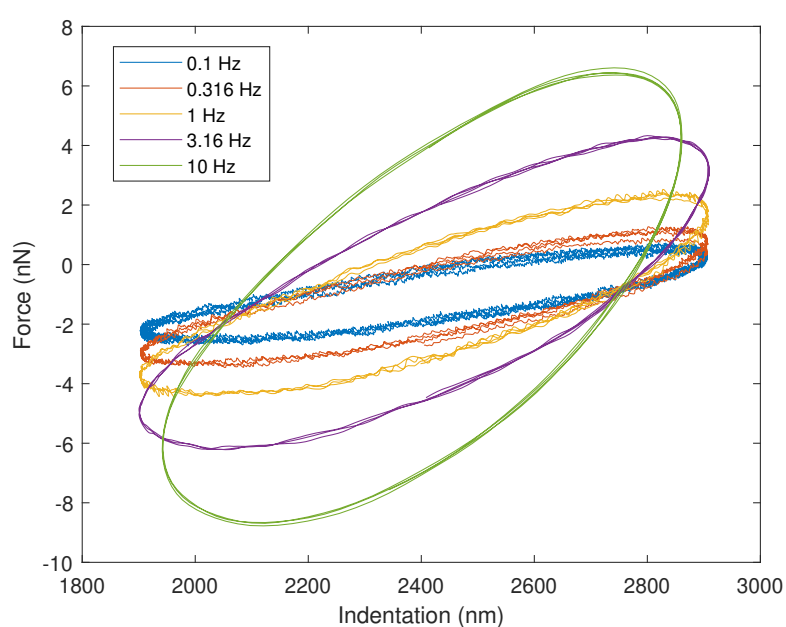

Figure 14: Force indentation responses from DMA on F11 cells at $0.1,0.316,1,3.16$ and $10 \mathrm{~Hz}$.

Of the studied nine cells, the Young's moduli at 3 and $4 \mu \mathrm{m}$ fell within the $10 \%$ of the initial value at $2 \mu \mathrm{m}$. A fixed indentation of $3 \mu \mathrm{m}$ was thus chosen to compare electrophysiological signals of F11s under mechanical loading. Indentationcontrolled feedback loop ensured that this indentation was held constant throughout the experiments.

\subsection{DMA fitting}

DMA allows for the identification of the complex modulus $E=E^{\prime}+i E^{\prime \prime}$, where $E^{\prime}$ and $E^{\prime \prime}$ are the storage and loss moduli, respectively. These moduli are a priori frequency dependent, i.e., the elastic and viscous behaviours of the material vary with the loading rate. To evaluate the evolution of moduli with frequency $f$, F11 cell bodies were indented at constant oscillating amplitude and logarithmic spaced frequencies: $0.1,0.316,1,3.16$ and $10 \mathrm{~Hz}$, recording larger force and stiffer responses at higher frequencies as shown in Figure 14. The moduli can then be fit against the experimental force $F v s$. indentation $h$ responses at each frequency through the following relations [61]:

$$
\begin{aligned}
& \frac{E^{\prime}(\omega)}{\left(1-v^{2}\right)}=\frac{F}{h} \cos (\delta) \frac{1}{2 \sqrt{h_{s} R}} \\
& \frac{E^{\prime \prime}(\omega)}{\left(1-v^{2}\right)}=\frac{F}{h} \sin (\delta) \frac{1}{2 \sqrt{h_{s} R}}
\end{aligned}
$$

where $v \sim 0.5, \delta$ is the phase lag between the indentation and force responses, $R$ is the nanoindenter probe radius, $h_{s}$ the static indentation depth and $\omega=2 \pi f$.

Noting that the relation between Young's modulus and shear modulus $G$ follows:

$$
G=\frac{E}{2(1+v)}
$$

one then obtains:

$$
\frac{G^{\prime}(\omega)}{(1-v)}=\frac{F}{h} \cos (\delta) \frac{1}{4 \sqrt{h_{s} R}}
$$

\begin{tabular}{c||c|c|c} 
AP feature & Before & During & After \\
\hline AP amplitude $(\mathrm{mV})$ & $65.34 \pm 12.52$ & $60.95 \pm 10.54$ & $64.36 \pm 11.99$ \\
AP width $(\mathrm{ms})$ & $36.85 \pm 13.78$ & $42.53 \pm 17.74$ & $37.78 \pm 14.84$ \\
$V_{\text {res }}(\mathrm{mV})$ & $-44.71 \pm 2.47$ & $-44.93 \pm 3.13$ & $-47.07 \pm 3.55$ \\
$V_{\text {th }}(\mathrm{mV})$ & $-28.55 \pm 5.85$ & $-28.41 \pm 6.54$ & $-30.63 \pm 6.39$ \\
AP rising phase $(\mathrm{ms})$ & $49.87 \pm 12.81$ & $52.38 \pm 15.28$ & $51.15 \pm 13.99$ \\
AP falling phase $(\mathrm{ms})$ & $131.92 \pm 33.91$ & $60.95 \pm 145.25$ & $144.48 \pm 35.46$ \\
Max $\mathrm{d} V_{m} / \mathrm{dt}(\mathrm{mV} / \mathrm{ms})$ & $1.72 \pm 0.31$ & $1.35 \pm 0.23$ & $1.67 \pm 0.17$ \\
AP firing freq $(\mathrm{APs} / \mathrm{s})$ & $0.15 \pm 0.10$ & $0.17 \pm 0.13$ & $0.19 \pm 0.20$
\end{tabular}

Table 3

Original analysed mean and SD AP features values before, during and after quasi-static indentation.

$$
\frac{G^{\prime \prime}(\omega)}{(1-v)}=\frac{F}{h} \sin (\delta) \frac{1}{4 \sqrt{h_{s} R}}
$$

\subsection{Original AP features values}

Table 3 includes the original mean values of the analysed AP features. From these, the normalised ones shown in Figures 8 and 9 are obtained by dividing each averaged value of interest in table 3 by its counterpart before indentation.

\subsection{Generalised Maxwell model}

The frequency-dependent mechanical response of viscoelastic materials (Section 7.6) can be modelled with the Generalised Maxwell model, which consists of a long-term elastic (spring) branch, in parallel with a set number of viscous branches (spring and dashpot) in series. Each viscous branch $i$ represents one relaxation response of the material, where relaxation time is defined as $\tau_{i}=\frac{\eta_{i}}{G_{i}}, G_{i}$ being the shear modulus (the spring) and $\eta_{i}$ being the viscosity (the dashpot) of the branch. The elastic branch, with a shear modulus $G_{\infty}$, corresponds to the elastic response when the viscous branches are not loaded elastically, e.g., after a relaxation time much larger than all $\max _{i}\left(\tau_{i}\right)$.

Using Prony series notation, the storage and loss moduli of a three-branch Maxwell model are then given by:

$$
\begin{aligned}
& G^{\prime}(\omega)=G_{0}\left[1-\sum_{i=1}^{3} g_{i}\right]+G_{0} \sum_{i=1}^{3} \frac{g_{i} \tau_{i} \omega^{2}}{1-\tau_{i}^{2} \omega^{2}} \\
& G^{\prime \prime}(\omega)=G_{0} \sum_{i=1}^{3} \frac{g_{i} \tau_{i} \omega^{2}}{1-\tau_{i}^{2} \omega^{2}}
\end{aligned}
$$

where $g_{i}=G_{i} / G_{0}$ and $G_{0}=G_{\infty}+\sum_{i} G_{i}$.

The parameters $G_{0}, g_{i}$ and $\tau_{i}$ were then fitted to match storage and loss moduli of the experimental DMA results by using a nonlinear least squares algorithm in MATLAB. They were then used as input in ABAQUS to define the 3D cell mechanical properties of the three-branch Maxwell model, see Table 4.

\subsection{D FE simulation}

The ABAQUS model consists of three distinct parts: the glass indenter, the cell and the glass bottom dish. The glass 
Action potential alterations induced by single F11 neuronal cell loading

\begin{tabular}{c|c} 
Magnitude & Value \\
\hline$G_{0}$ & $479.99 \mathrm{~Pa}$ \\
$g_{1}$ & 0.06 \\
$\tau_{1}$ & $2.50 \mathrm{~s}$ \\
$g_{2}$ & 0.09 \\
$\tau_{2}$ & $211.77 \mathrm{~ms}$ \\
$g_{3}$ & 0.82 \\
$\tau_{3}$ & $9.85 \mathrm{~ms}$
\end{tabular}

Table 4

Calibrated viscoelastic parameters.

indenter was modelled as a spherical shell with an outer radius of $3 \mu \mathrm{m}$. The indenter was meshed with quadrilateral shell elements and assumed quasi-rigid. The 3D cell geometry was constructed from a z-stack'ed widefield image of a live F11 cell. The geometry was smoothed, meshed with tetrahedral elements, and imported into ABAQUS. The cell was modelled as a linear viscoelastic material whose mechanical properties were taken directly from the DMA results (with $v=0.49$ ). The cell-culture dish was modelled as a rigid plane and was spatially fixed in the simulation. An interaction algorithm was defined between the indenter and the cell, as well as between the cell and the plate, in which a 'hard' contact was chosen for the normal direction, coupled to a penalty friction formulation with a friction coefficient of 0.2. A general static simulation was run in which a $3 \mu \mathrm{m}$ downward displacement was applied to the indenter in $1 \mathrm{~s}$, and sustained for another $20 \mathrm{~s}$ (thus longer than the relaxation period observed experimentally). The surface area of the cell both before and during the indentation was finally extracted for comparison.

\subsection{Electrophysiological model}

The parameters and channel gating evolution needed to model the AP through Equation (1) were taken from the DRG model in Ref. [26]. The model parameters are given in Table 5, while the potassium and sodium gating variables evolution equations are given in Tables 6 and 7, respectively.

The analysis of the AP features predicted by Equation (1) for a $10 \%$ area increase (i.e., the area increase needed to fit the experimental results, see Section 4.1) is given in Figure 15. The results of a $40 \%$ increase are also given for illustration.

\begin{tabular}{c|c} 
Magnitude & Value \\
\hline$C_{m}$ & $4.43 \times 10^{-11} \mathrm{~F} / \mathrm{m}$ \\
$\bar{G}_{N a}$ & $1.41 \times 10^{-6} \mathrm{~S} / \mathrm{m}$ \\
$\bar{G}_{N a_{s}}$ & $2.76 \times 10^{-7} \mathrm{~S} / \mathrm{m}$ \\
$\bar{G}_{k}$ & $8.40 \times 10^{-8} \mathrm{~S} / \mathrm{m}$ \\
$G_{l}$ & $5.60 \times 10^{-9} \mathrm{~S} / \mathrm{m}$ \\
$V_{m}$ & $-49.1 \mathrm{mV}$ \\
$E_{N a}$ & $62.94 \mathrm{mV}$ \\
$E_{K}$ & $-92.34 \mathrm{mV}$ \\
$E_{l}$ & $-54.3 \mathrm{mV}$ \\
$A_{0}$ & $1256.64 \mu \mathrm{m}^{2}$ \\
$A$ & $1261.66 \mu \mathrm{m}^{2}$
\end{tabular}

Table 5

Parameter values used in the DRG electrophysiological model.

$$
\begin{gathered}
\text { Potassium channel } \\
\frac{d n}{d t}=\alpha_{n}(V)(1-n)+\beta_{n}(V) n \\
\alpha_{n}(V)=0.001265 \frac{V+14.273}{1-e^{\frac{V+14.273}{-10}}} \\
\beta_{n}(V)=0.125 e^{\frac{V+55}{-2.5}}
\end{gathered}
$$

Table 6

Potassium channels' gating equations.

$$
\begin{array}{c|c}
\text { Sodium channel } & \text { Slow-inactivated sodium channel } \\
\hline \frac{d m}{d t}=\alpha_{m}(V)(1-m)+\beta_{m}(V) m & \frac{d m_{s}}{d t}=\alpha_{m_{s}}(V)\left(1-m_{s}\right)+\beta_{m s}(V) m_{s} \\
\frac{d h}{d t}=\alpha_{h}(V)(1-h)+\beta_{h}(V) h & \frac{d h_{s}}{d t}=\alpha_{h_{s}}(V)\left(1-h_{s}\right)+\beta_{h_{s}}(V) h_{s} \\
\alpha_{m}(V)=\frac{11.49}{1+e^{\frac{V+8.58}{-8.47}}} & \alpha_{m_{s}}(V)=\frac{1.032}{1+e^{\frac{V+1.699}{-14.87115}}} \\
\beta_{m}(V)=\frac{11.49}{1+e^{\frac{V+67.2}{27.8}}} & \beta_{m_{s}}(V)=\frac{5.79}{1+e^{\frac{V+130.4}{22.9}}} \\
\alpha_{h}(V)=0.0658 e^{\frac{-(V+120)}{20.33}} & \alpha_{h_{s}}(V)=\frac{0.06435}{1+e^{\frac{V+73.26415}{3.71928}}} \\
\beta_{h}(V)=\frac{3.0}{1+e^{\frac{V-6.8}{-12.998}}} & \beta_{h_{s}}(V)=\frac{0.13496}{1+e^{\frac{V+10.027853}{-9.09334}}}
\end{array}
$$

Table 7

Sodium channels' gating equations. 

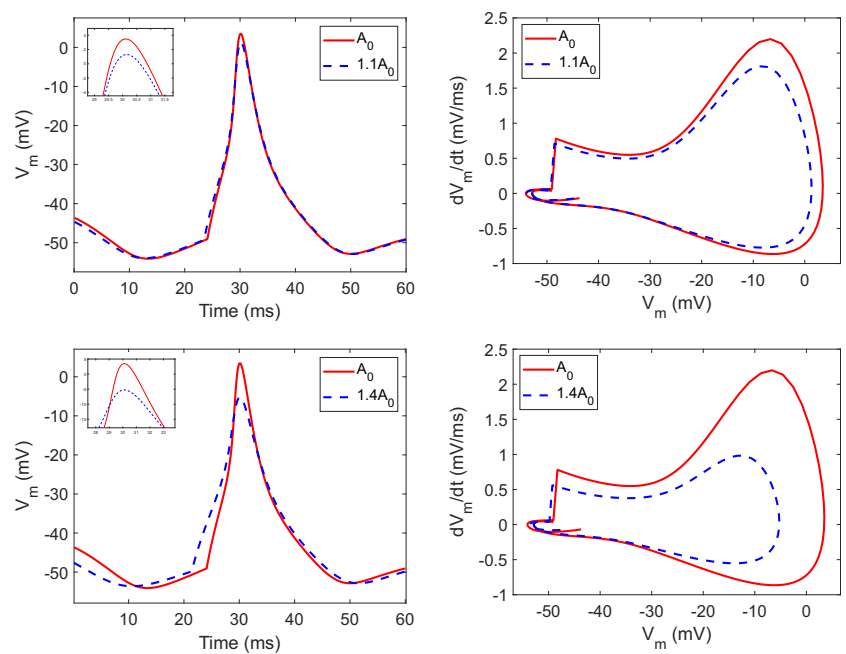

Figure 15: Left: prediction of APs through Equation (1) before and during a $10 \%$ (above) and $40 \%$ (below) area increase, as a result of cell loading during indentation. Insets: AP peaks zoomed in. Right: phase plots of the APs. 